\title{
A novel 3D surface generation model for micro milling based on homogeneous matrix transformation and dynamic regenerative effect
}

\author{
Wanqun Chen ${ }^{1,2}$, Wenkun $\mathrm{Xie}^{2}$, Dehong Huo ${ }^{1^{*}}$, Kai Yang ${ }^{3}$ \\ 1. Mechanical Engineering, School of Engineering, Newcastle University, Newcastle upon Tyne, NE1 \\ $7 R U, U K$ \\ 2. Center for Precision Engineering, Harbin Institute of Technology, Harbin 150001, P. R. China \\ 3. Army Aviation Institute, Beijing 101123, P. R. China
}

${ }^{*}$ Corresponding author: D. Huo, Tel: 44 (0) 191208 6230, E-mail: dehong.huo@newcastle.ac.uk

\begin{abstract}
A novel 3D surface generation modelling method for micro milling which considers the effect of machining nonlinear dynamics is proposed based on homogeneous matrix transformation. Considering the effects of machining process kinematics, tool run-out and the nonlinear dynamic regenerative effect of the machining system, the relationship between machining process and surface topography is established. On this basis, three typical machining cases, namely static stable, dynamic stable and unstable machining, are investigated in this paper. The machining experiment results demonstrate that the proposed surface generation model can accurately predict surface topography generation and roughness values with different machining conditions. Thus, it can be used not only to optimize the machining parameters for improving the micro-milled surface quality but also to predict the surface topography under pre-defined machining parameters.
\end{abstract}

Keywords: Surface generation modelling; homogeneous matrix transformation; micro milling; nonlinear dynamic; dynamic regenerative effect

\section{Introduction}

Micro milling is recognized as a versatile micro manufacturing process to achieve $3 \mathrm{D}$ micro features and has been widely applied in micro-components fabrication, such as micro-chemical devices, bio-sensors and micro moulds $[1,2]$. The quality of the machined surface is mainly determined by their surface roughness and topography. While, as devices and their features are increasingly miniaturized, the surface area to volume ratio tends to increase [3]. Thus, the surface roughness and texture of machined surface have an important effect on their performance $[4,5]$. In micro milling, poor surface quality is becoming a significant technical obstacle preventing the widespread use of micro milling in some applications. For the improvement of surface quality of the micro milled surface, accurate modeling and predication on the surface generation in micro milling, especially establishing the relationship between the machining process and the surface generation, is crucial.

The surface generation in micro milling is a complicated process and it is easily affected by many factors, including material elastic recovery, cutter geometry, machining parameters, and nonlinear dynamics of the machine tool system, etc. To improve the quality of the micro-machined surface, it is necessary to investigate the effects of process parameters and the nonlinear dynamics in the machining process on the surface generation. 
Numerous researches have been recently carried out to investigate the nonlinear dynamics and surface generation separately in micro milling. For the investigation of nonlinear dynamics in milling process, Govekar et al. [6] proposed a semi-discretization method to investigate the stability and dynamics in the milling process at small radial depth of cut, quasiperiodic chatter and periodic chatter are predicted. By using a set of delay differential equations with periodic coefficients to describe the system dynamics, Long et al. [7] studied the dynamics of milling processes with variable time delays, focusing on the influence of the feed rate and the loss-of contact effect. In order to improve the calculation accuracy, and shorten the calculation time, Li et al. [8] presented a complete discretization scheme for milling stability prediction. In their model all parts of delay differential equation are discretized by using the numerical iteration method. Afazov et al. [9] proposed a chatter modelling in micro-milling by taking into account the nonlinearity of the uncut chip thickness including the run-out effect and the dynamics of the tool-holder-spindle assembly. In the present, the nonlinear dynamics and the chattering performance in micro milling are well studied, which is meaningful for the machining parameters selection. However, the information of the surface topography cannot be obtained from such models. To investigate the surface topography in micro milling process, various numerical or geometric models have been proposed to describe the surface generation process. In those numerical models, tool and workpiece are represented by discrete points, and on this basis of the relative motion relationship between tool and workpiece, the surface topography is finally fitted. While, the geometric model is established based on the volume oriented constructive solid geometry (CSG) method, which can be applied to create a complex surface or object by using Boolean operators to combine simpler objects [10]. Peigne et al. [11] performed the 2.5D geometrical modelling of the milled surface generation by using a time domain simulation of end milling operations, where a quadratic form to interpolate the cutting edge path made it possible to obtain a small interpolation error even if angular steps are roughly cut. Based on the minimum chip thickness concept. Vogler et al. and Sun et al. [12,13] developed a surface generation model to predict the surface roughness of the slot floor centerline, and the relationship among surface roughness, feed per tooth, and the cutter geometry were investigated. Chen et al. [14] investigated the influence of the tool runout, minimum chip thickness as well as the tool geometric parameters on the surface generation, but the simulation was only conducted in a 2D model. By using kinematic rules and transformation operators, Kouravand et al. [15] established a microchannel surface texture model which is based on CSG method, considering the geometrical features of cutting edge and minimum chip thickness. Li et al. [16] proposed a trajectory-based surface roughness model for micro end milling to capture the role of minimum chip thickness, micro tool geometry and process parameters in micro-milling process. Also, by taking the material removal volume and cutting velocity into account, a surface roughness model with tool wear effect was also developed.

To summarize, in most of the present surface generation models for end milling process, the topographies of the machined surface were simplified into a 2D profile of slot floor centerline. Due to the cycloid trajectory in micro milling process, the whole topography of the machined surface cannot be fully reflected by the profile of slot floor centerline. Although there are a few efforts made on 3D modeling of end milling processes, the influence of the nonlinear dynamic response in machining process and surface generation was not fully considered, which affects the accuracy of these surface generation simulations.

For the reasons above, a homogeneous matrix transformation (HMT) based real 3D surface generation model is proposed to consider the effects of tool run-out and dynamics of the machine tool system on the cutter trajectories. Meanwhile, based on the influence of the dynamic performance of the 
machining system on the surface generation, the dynamic regenerative effect in milling process is also investigated, thereby establishing a link between machining process and surface generation.

\section{Surface generation model based on HMT considering machining dynamics}

The proposed simulation method for surface generation is outlined in Fig.1. In most cases, the surface generation process is easily influenced by machining system. Moreover, the machining of workpiece is realized by the kinematic movements of the slides and spindle of the machine tool, which are controlled by the machining program. Thus, to accurately describe the surface generation process, first, the machining program should be well pre-defined, according to the realistic targeted shape of workpiece.

Further, in micro milling operation, due to the high rotational speed, the manufacturing errors and alignment errors of the milling tool cannot be neglected. The dynamic tool run-out can be recognized as the hybrid results of those errors [17]. So the effect of the manufacturing errors and alignment errors can be considered by involving the dynamic tool run-out in the modeling of surface generation.

Since the machining system is not a rigid body system, the cutting force generated during the milling process could induce the relative displacement between tool and workpiece, which in turn changes the ideal tool trajectory. Thus, it is necessary to investigate effects of the tool run-out and the dynamic response of the machining system on the surface generation in micro milling. In the proposed method, the dynamic performance of the milling machining system that includes the workpiece, machine tool and micro cutter, are obtained by FE simulation or experimental test. The tool tip trajectory is calculated including the influence from the tool flexibility and the dynamic response of the machining system. However, the cutting edge is modelled as rigid, thus all the points on cutting edge have the same trajectory with the tool tip. The whole machining process is simulated by using HMT.

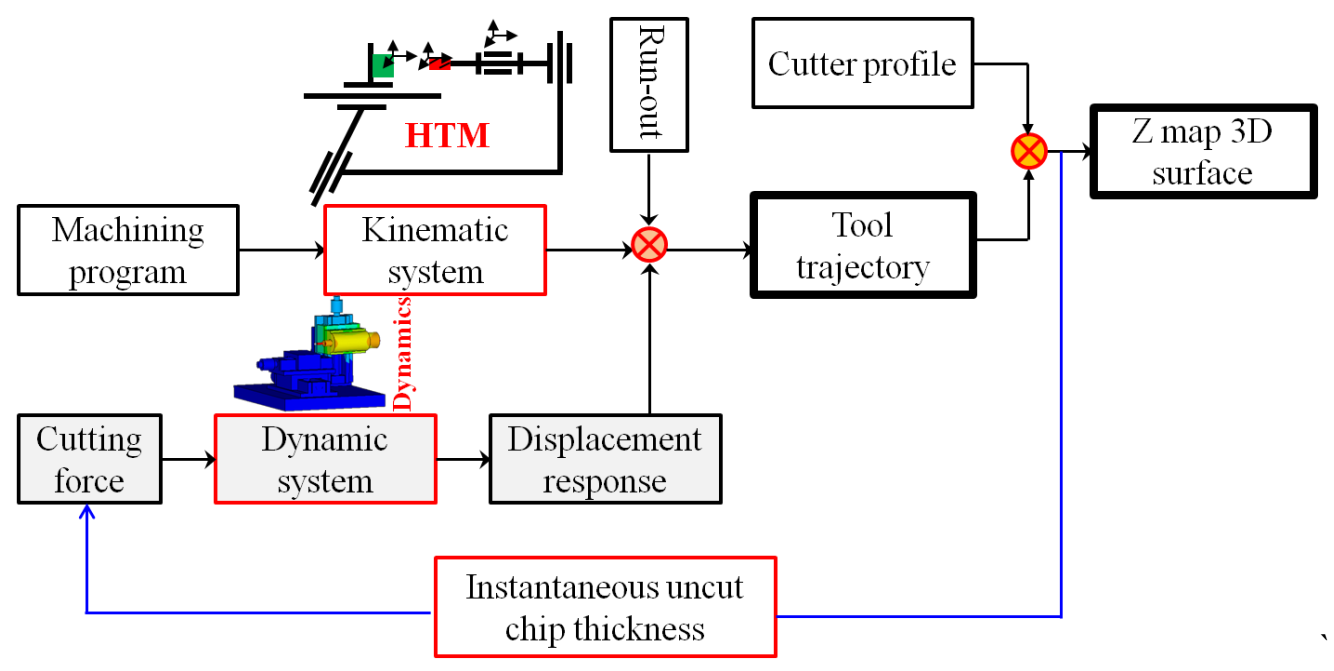

Fig.1 Outline of the proposed surface simulation method

By considering the abovementioned factors, tool trajectory is updated in a specific time interval. The cutter profile sweeps along the updated tool trajectory to generate the actual cutting path, and then the $\mathrm{Z}$ map technology is adopted to generate the final machine surface [18].

\subsection{Establishment of HTM in end milling process}

\subsubsection{Definition of each coordinate system}


As illustrated in Fig. 2 a), four coordinate systems and their transformation matrixes are defined in the model.

a)

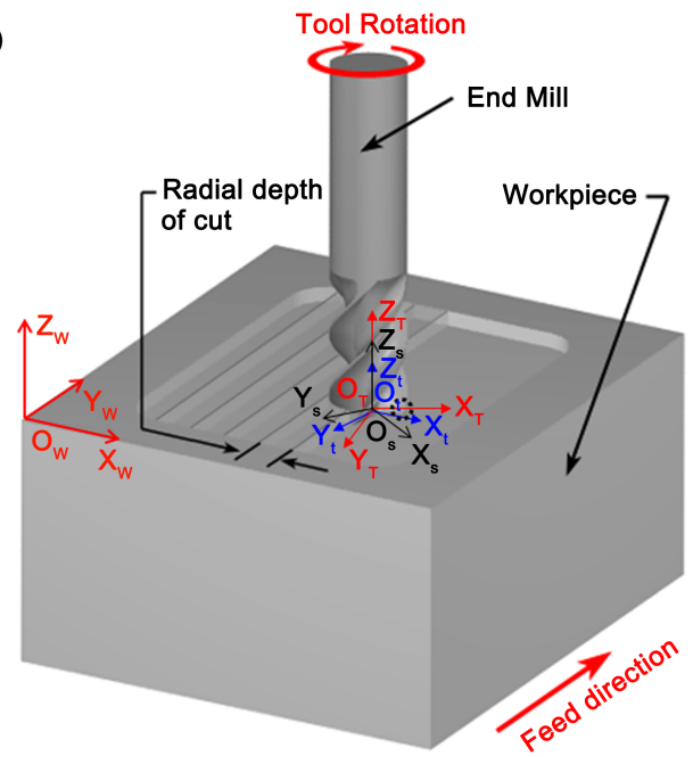

b)

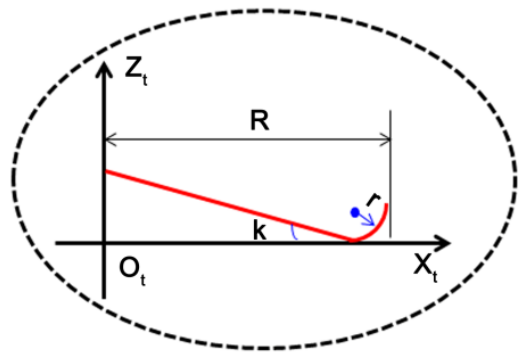

$z=\left\{\begin{array}{c}-x \tan k+(R-r-r \sin k) \tan k+r(1-\cos k) \\ -\sqrt{r^{2}-[x-(R-r)]^{2}}+r\end{array}\right.$

Fig.2 Coordinate systems and tool edge profile

\subsubsection{Local coordinate system of tool cutting edge $\left(O_{t}-X_{t} Y_{t} Z_{t}\right)$}

$O_{t}-X_{t} Y_{t} Z_{t}$ is the local coordinate system of the tool and its coordinate origin is set at the end of the tool center. The cutter edge in $O_{t}-X_{t} Y_{t} Z_{t}$ is shown in Fig.2 (b). It can be depicted by the following equation:

$$
z=f(x)=\left\{\begin{array}{cc}
-x \tan k+(R-r-r \sin k) \tan k+r(1-\cos k), & 0<x<R-r-r \sin k \\
-\sqrt{r^{2}-[x-(R-r)]^{2}}+r, & x \geq R-r-r \sin k
\end{array}\right.
$$

where $r, R$, and $k$ denotes the corner radius, radius and rake angle of the tool, respectively.

The coordinates of the cutting edge in $O_{t}-X_{t} Y_{t} Z_{t}$ is given as:

$$
\left[\begin{array}{c}
x_{t} \\
y_{t} \\
z_{t} \\
1
\end{array}\right]=\left[\begin{array}{c}
x \\
0 \\
f(x) \\
1
\end{array}\right]
$$

\subsubsection{Coordinate system of tool $\left(O_{T}-X_{T} Y_{T} Z_{T}\right)$}

The establishment of the $O_{T}-X_{T} Y_{T} Z_{T}$ is to consider the rotation movement of the tool around the spindle axis and the translation movement relative to the workpiece. As given in Fig.2, $X_{T}$ and $Y_{T}$ axes are aligned with feed direction and cross-feed direction, $Z_{T}$ axis is parallel to $Z_{t}$. The origin of the tool coordinate system is the same as that of the local tool coordinate system. The angular velocity of $O_{t^{-}}$ $X_{t} Y_{t} Z_{t}$ relative to $O_{T}-X_{T} Y_{T} Z_{T}$ is equal to the spindle angular velocity, the relative rotational angle $\theta$ can be expresses as:

$$
\theta=\varphi-\omega t
$$

where $\varphi$ is the initial angle between $X_{t}$ and $X_{T}$ axes and it is set to $0^{\circ}$ in this paper. $\omega$ is tool rotation angular velocity $(\mathrm{rad} / \mathrm{s}), t$ is cutting time $(s)$.

The HTM from $O_{t}-X_{t} Y_{t} Z_{t}$ to $O_{T}-X_{T} Y_{T} Z_{T}$ can be expressed as: 


$$
{ }^{T} T_{t}=\left[\begin{array}{cccc}
\cos \theta & -\sin \theta & 0 & 0 \\
\sin \theta & \cos \theta & 0 & 0 \\
0 & 0 & 1 & 0 \\
0 & 0 & 0 & 1
\end{array}\right]
$$

\subsubsection{Coordinate system of spindle $\left(O_{S}-X_{S} Y_{S} Z_{S}\right)$}

The $O_{S}-X_{S} Y_{S} Z_{S}$ is established to describe the relative translation of the spindle and workpiece. By transforming $O_{T}-X_{T} Y_{T} Z_{T}$ to $O_{S}-X_{S} Y_{S} Z_{S}$, the spindle run-outs along axial and radial directions can be considered. The amplitude and initial phase angle of radial run-out are defined to $\Delta d$ and $\Delta \alpha$. And those of axial run-out are $\Delta h$ and $\Delta \beta$. Therefore, the HTM from $O_{T}-X_{T} Y_{T} Z_{T}$ to $O_{S}-X_{S} Y_{S} Z_{S}$ can be expressed as:

$$
{ }^{s} T_{T}=\left[\begin{array}{cccc}
1 & 0 & 0 & \Delta d \sin (\Delta \alpha-\omega t) \\
0 & 1 & 0 & \Delta d \cos (\Delta \alpha-\omega t) \\
0 & 0 & 1 & \Delta h \sin (\Delta \beta-\omega t) \\
0 & 0 & 0 & 1
\end{array}\right]
$$

\subsubsection{Coordinate system of workpiece $\left(O_{W}-X_{W} Y_{W} Z_{W}\right)$}

$O_{W}-X_{W} Y_{W} Z_{W}$ is defined as the coordinate system of the workpiece. By transformation of the coordinates of the tool cutting edge from $O_{t}-X_{t} Y_{t} Z_{t}$ to $O_{W}-X_{W} Y_{W} Z_{W}$, the relative position of any points on the cutting edge to workpiece can be obtained. And the height of the cutting edge at any point can be recorded through the $Z_{W}$ coordinates.

For each feed in cross-feed direction, in the $O_{W}-X_{W} Y_{W} Z_{W}$, the origin of $O_{S}-X_{S} Y_{S} Z_{S}$ can be depicted by:

$$
\left\{\begin{array}{c}
x_{0 S}=x_{0}+(i-1) a_{e} \\
y_{0 S}=y_{0}+v_{f} t \\
z_{0 S}=z_{0}
\end{array}\right.
$$

where $\left(x_{0}, y_{0}, z_{0}\right)$ is the initial origin of $O_{S} X_{S} Y_{S} Z_{S}$ in the $O_{W}-X_{W} Y_{W} Z_{W}$, and it is set as $(0,-R, 0)$ in this paper; $a_{e}$ is the radial cutting depth $(\mathrm{mm}) ; i$ is the number of feed times in cross-feed direction; $v_{f}$ is the feed speed in $Y_{w}$ direction $(\mathrm{mm} / \mathrm{s})$.

\subsection{Establishment of homogenous transformation matrix}

As shown in Fig.1, owing to the dynamic response of the cutting tool, the relative displacement between tool and workpiece is changed. For each feed in radial direction, the coordinates of the origin of the $O_{S}-X_{S} Y_{S} Z_{S}$ relative to those of $O_{W}-X_{W} Y_{W} Z_{W}$ can be expressed as:

$$
\left\{\begin{array}{c}
x_{0 S}=x_{0}+(i-1) a_{e}+x(t) \\
y_{0 S}=y_{0}+v_{f} t+y(t) \\
z_{0 S}=z_{0}
\end{array}\right.
$$

where $x(t)$ and $y(t)$ denote the dynamic instantaneous displacement between the tool and workpiece in $x$ and $y$ direction.

The HTM from $O_{S}-X_{S} Y_{S} Z_{S}$ to $O_{W}-X_{W} Y_{W} Z_{W}$ can be expressed as: 


$$
{ }^{W} T_{S}=\left[\begin{array}{cccc}
1 & 0 & 0 & x_{0}+(i-1) a_{e}+x(t) \\
0 & 1 & 0 & y_{0}+v_{f} t+y(t) \\
0 & 0 & 1 & z_{0} \\
0 & 0 & 0 & 1
\end{array}\right]
$$

In the $O_{W} X_{W} Y_{W} Z_{W}$, the cutting edge equation can be expressed as:

$$
\left[\begin{array}{c}
x_{W} \\
y_{W} \\
z_{W} \\
1
\end{array}\right]={ }^{W} T_{S}{ }^{S} T_{T}{ }^{T} T_{t}\left[\begin{array}{c}
x_{t} \\
y_{t} \\
z_{t} \\
1
\end{array}\right]=\left[\begin{array}{c}
x \cos \theta+\Delta d \cos (\Delta \alpha-\omega t)+x_{0}+(i-1) a_{e}+x(t) \\
x \sin \theta+\Delta d \sin (\Delta \alpha-\omega t)+y_{0}+v_{f} t+y(t) \\
f(x)+\Delta h \sin (\Delta \beta-\omega t)+z_{0} \\
1
\end{array}\right]
$$

The $\mathrm{Z}$ map technology is adopted to depict the cutting trajectories in the central line. When the tool run-out and dynamic displacements are comparable with feed pert tooth, the micro milling process may become instable. In such case, owing to the effects of the machine tool vibration or tool run-out, in an instantaneous cutting path, e.g. the $(n+1)^{\text {th }}$ cutting path, the tool edge may pass through workpiece without removing any material. The machined surface may also be cut again by the following cutting path, e.g. the $(n+3)^{\text {th }}$ cutting path, as shown in Fig.3. Thus, for a specific point $(x, y)$ on the workpiece, the minimum value of the $\mathrm{z}$ coordinate is adopted to form the final surface of the workpiece.

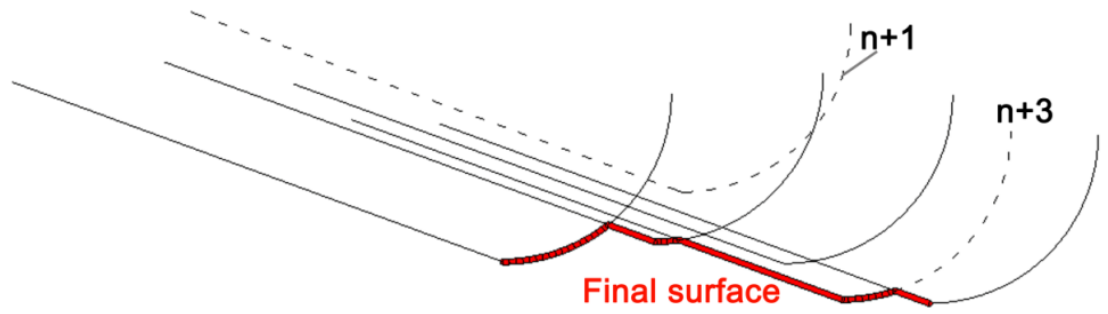

Fig.3 Diagram for tool path in centre line of slot milling

According to the copy principle of the tool contour, the surface topography of the workpiece is formed by the sweeping of the cutting edge profile along the tool path. To model surface generation through numerical simulation, the tool cutting edge and workpiece are discretized into a series of elements. The tool edge contour is mapped onto the workpiece surface by comparison and substitution of the corresponding points to form the machined surface topography.

\subsection{Establishment of surface generation simulation algorithm}

Fig. 4 illustrates the schematic diagram for the surface generation algorithm in the present work. Firstly, the cutting edge is discretized into many elements, and the workpiece is evenly divided into $n$ columns and $m$ rows. Each row and column are intersected at a grid point. By calculating the tool cutting depth (z-coordinate) at grid point, the height of each point on the machined surface of the workpiece can be obtained. And the height of the middle position between two grid points can be obtained by a curve fitting algorithm to form a complete surface. 


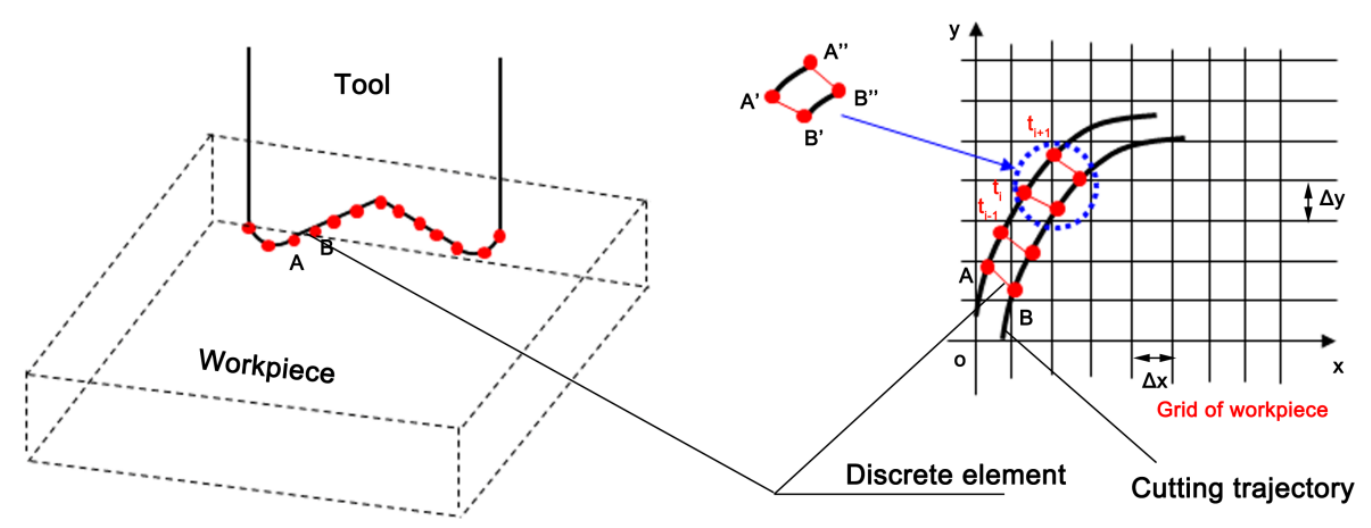

Fig.4 Schematic of surface generation algorithm

Fig. 5 shows the flowchart for the proposed surface generation simulation algorithm:

step 1: Select an appropriate discrete length $d$, to divide the workpiece into $m \times n$ grids, and set the initial height of each point as $a_{p}$.

step 2: Determine the discrete length of the tool $\Delta L$, and the time interval $\Delta t$. Calculate the cutting time $\mathrm{t}$ of each feed and feed times $\mathrm{n}$ according to the length and width of the workpiece, respectively.

step 3: Calculate dynamic displacement between tool and workpiece based on the machining parameters and the dynamic performance of the machine system.

step 4: Perform matrix transformation of each point on the cutter with the dynamic displacements obtained from step 3 in each time interval. Thus, the dynamic coordinates of each point in the cutting edge in workpiece coordinate system is obtained for each time interval according to the cutting edge equation.

step 5: The height of the discrete point of each cutting edge at each time interval obtained from step 4 is compared with the current value. If the height is less than the current height of the workpiece, it means that the tool is cut into the workpiece, and the height of the point on the cutting edge is used to replace of the current height of the workpiece. Otherwise the workpiece height is maintained at its original value.

step 6: According to the finial height of the corresponding workpiece, the three-dimensional machined surface is generated. The topography of the machined surface and the influence of the machining parameters on roughness can be predicted. 


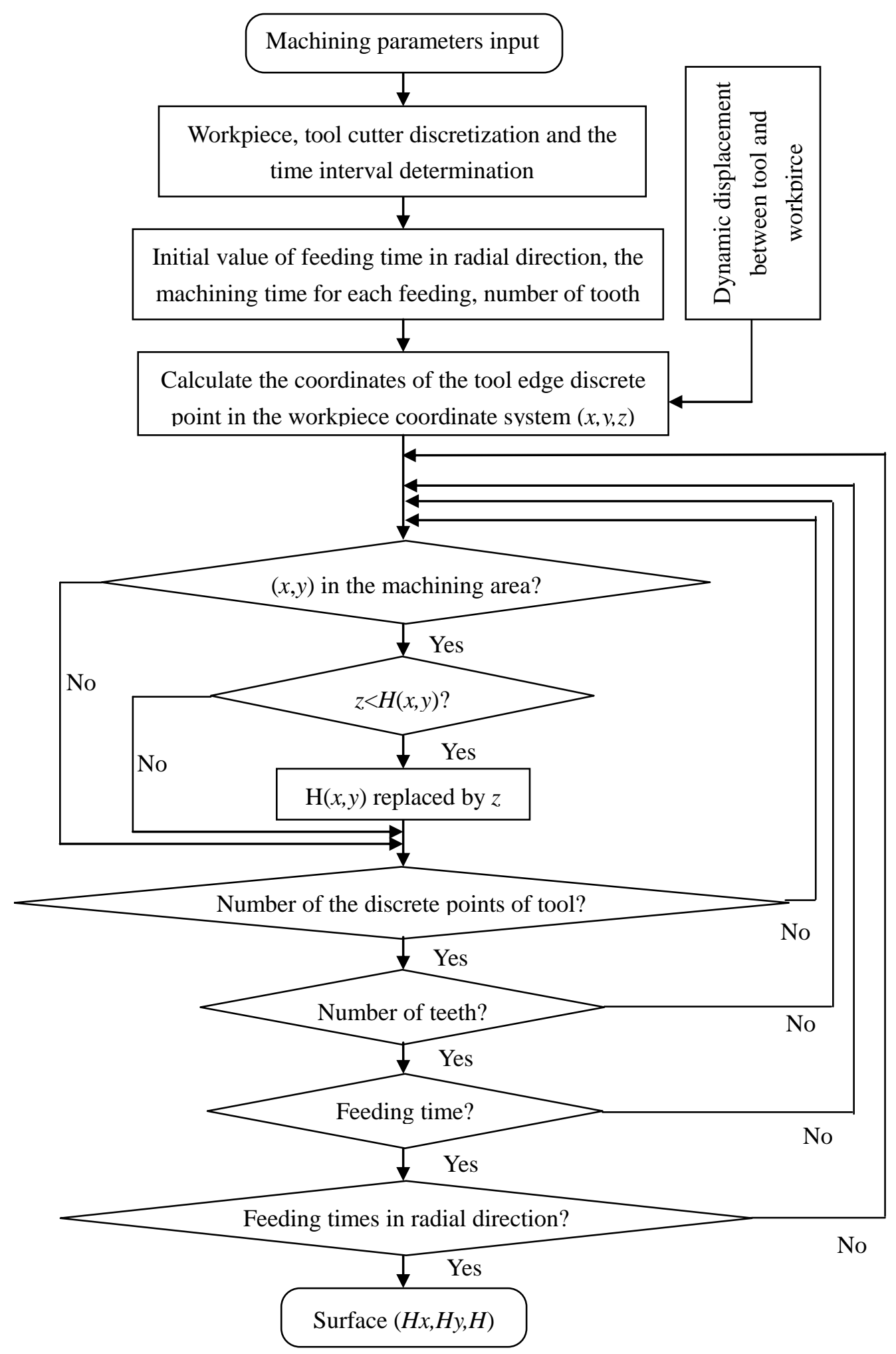

Fig.5 Flowchart of the surface generation algorithm in micro milling process

\section{Surface generation simulation and experimental verification}

In this section, the proposed method is applied for the modeling and predication of surface topography of the machined surface by micro milling. 


\subsection{Experimental setup}

To validate the surface generation model machining experiments are conducted on a 3-axis micro milling machine (Nanowave MTS5R). The machine tool is equipped with three precision linear stages which are driven by DC servo motors with smallest feed of $0.1 \mu \mathrm{m}$ and a high-speed spindle $(5,000$ $80,000 \mathrm{rpm}$ ). The experimental setup is presented in Fig. 6. A 3-component piezoelectric dynamometer (Kistler 9256C2) is mounted on the $\mathrm{X}-\mathrm{Z}$ stages to measure feed and cross-feed cutting forces. The workpiece is clamped on a fixture attached to the dynamometer. $1 \mathrm{~mm}$ diameter two-flute micro flat end mills are used in slot milling of Al6061.
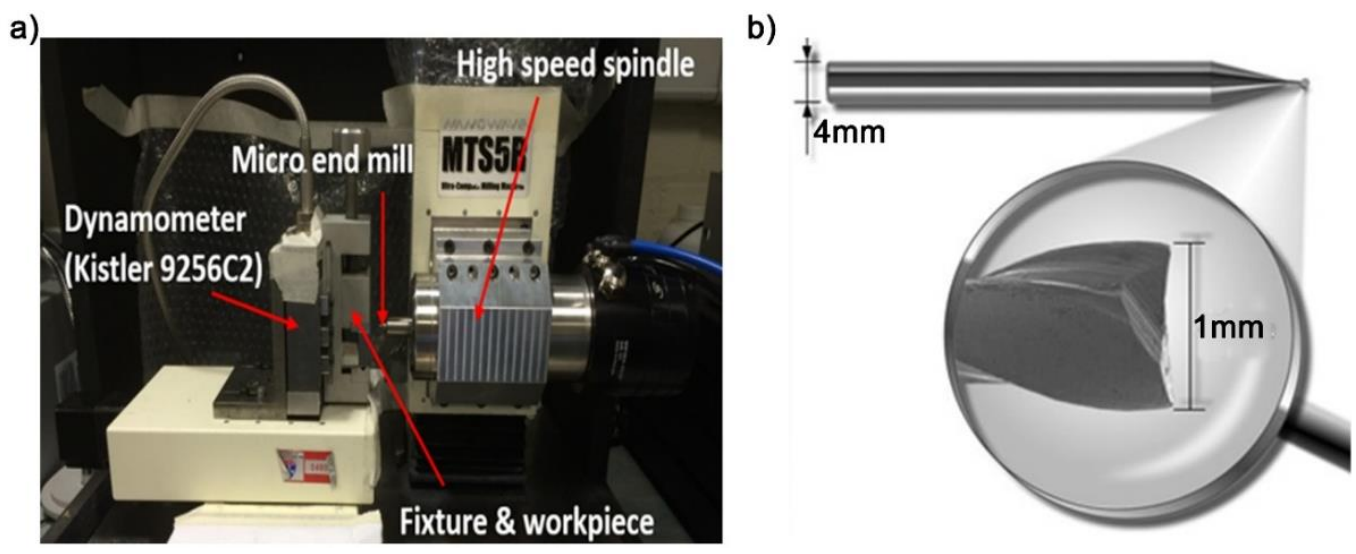

Fig.6 Experimental setup, (a) micro milling machine, (b) micro milling cutter

\subsection{Tool geometric parameter detection}

In order to obtain the geometry parameters, the micro end mills were examined by SEM prior to machining experiments. Fig.7 shows the side view and top view of the micro cutting tool. The geometrical parameters of the micro cutting tool are shown in Table 1.

Table 1 Parameters of the micro cutting tool

\begin{tabular}{ll}
\hline Parameter & Value \\
\hline Cutting diameter, $D$ & $1 \mathrm{~mm}$ \\
Number of flutes, $N$ & 2 \\
Cutting edge radius, $r_{e}$ & $3 \mu \mathrm{m}$ \\
Minor cutting edge angle, $k$ & $5^{\circ}$ \\
Corner radius, $r$ & $5 \mu \mathrm{m}$ \\
\hline
\end{tabular}

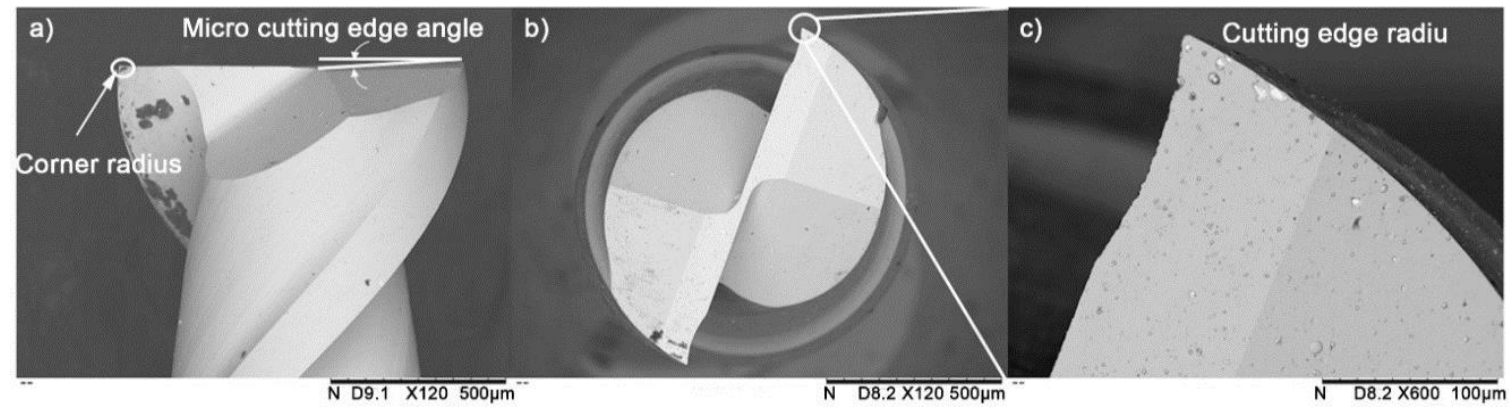

Fig.7 SEM images of the micro cutter tool used in the simulation and experiment. a) side view; b) top view; c) zoomed view.

\subsection{Machining dynamic analysis}




\subsubsection{Tool run-out identification}

In this experiment, the capacitive displacement sensor (Micro-epsilon, CS005) was used to realize the real-time measurements of the tool run-out. As given in Fig. 8 (a), the sensor probe was first mounted near the shaft. The distance between probe and shaft is should be within the measurement range of the probe. Then the shaft was rotated slowly to make sure that the probe is not contact with the shaft at its closest point. Fig.8 (b) shows the magnitude of the tool run-out and the related run-out angle with rotational speed of $15,000 \mathrm{rpm}, 60,000 \mathrm{rpm}$ and $80,000 \mathrm{rpm}$ respectively. The measured offset distance, $r_{0}$, is $0.5 \mu \mathrm{m}, 1.2 \mu \mathrm{m}$ and $2.3 \mu \mathrm{m}$, respectively.

a)

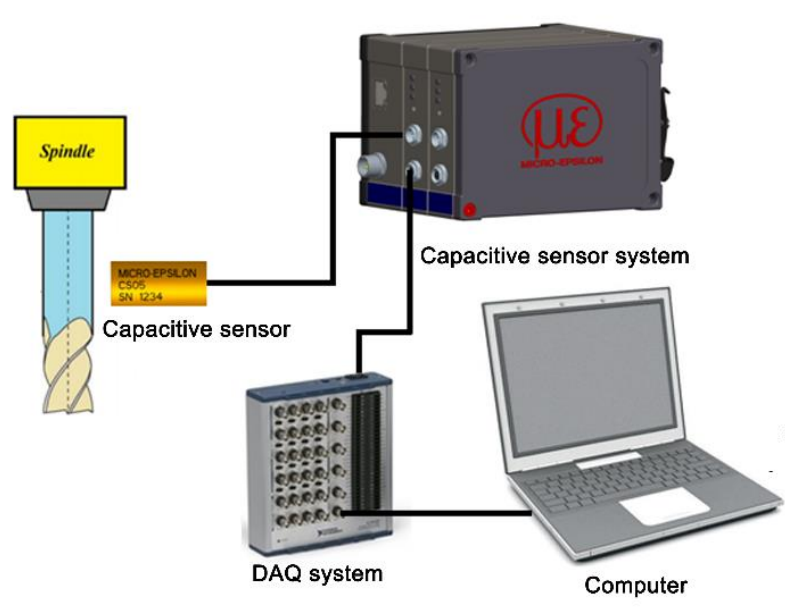

b)

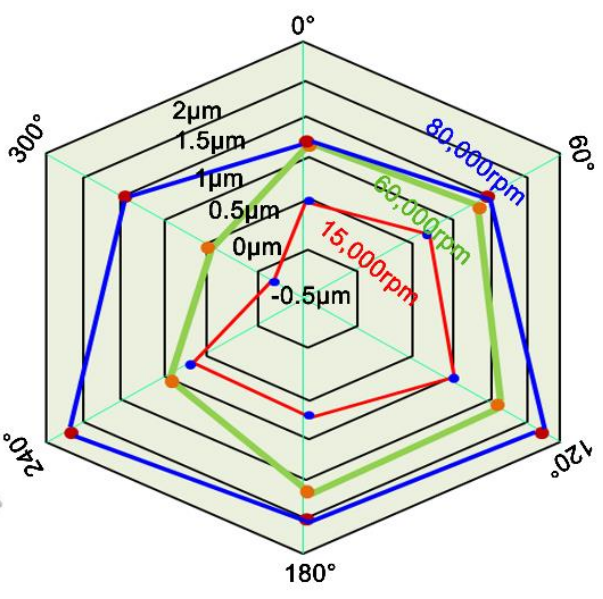

Fig. 8 (a) Schematic diagram of the experimental setup for tool run-out measurement, (b) Measured results of the tool run-out.

\subsubsection{Dynamic analysis of the machine system}

The dynamic performance of a machine system can be evaluated by experiments and finite element simulations. Due to the small diameter $(1 \mathrm{~mm})$ of the milling tool, it is difficult to experimentally detect the dynamic response of tool tip. Therefore, finite element simulations are adopted. Fig.9 a) shows the finite element model for the micro milling machine tool. The key input parameters of the machine tool components, such as bearing stiffness, materials properties, damping coefficients, are extracted from relevant datasheet or determined from dynamic tests. The contact rolling bearings in the slides and the spindle are modelled by spring elements, as shown in the inset of Fig. 9.

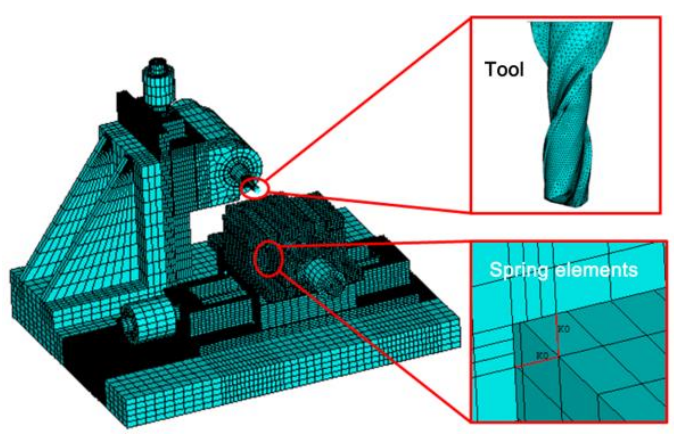

a)

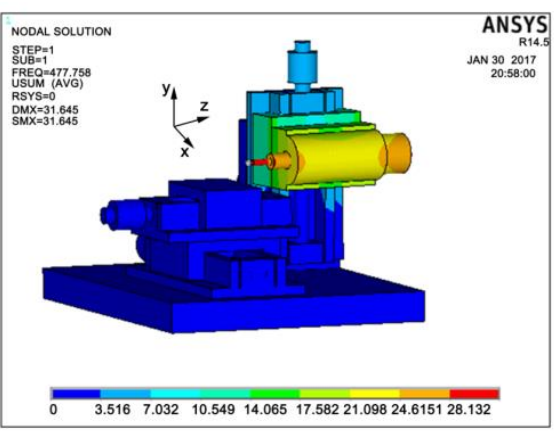

b)

Fig.9 Finite element model of the micro milling machine system

A modal analysis was first performed to obtain the natural frequencies of machine tool and the 
corresponding vibration modes. As shown in Fig.9b), the $1^{\text {st }}$ order natural frequency of the machine system is $478 \mathrm{~Hz}$ and its vibration mode is the spindle swinging around $\mathrm{y}$-axis with the Y slide.

Fig.10 shows the dynamic response of the tool in $x$ and $y$ directions. By using the peak-picking fitting method, the modal dynamic parameters are determined [20]. Then the modal dynamic parameters obtained by both methods are used to determine the chatter stability lobes.

The peak-picking fitting method can be described as follows. The FRF in the real and imaginary parts can be given by

$$
\begin{aligned}
& \operatorname{Re}(F R F)=\sum_{i=1}^{n} \frac{1}{k_{i}}\left(\frac{1-r_{i}^{2}}{\left(1-r_{i}^{2}\right)^{2}+\left(2 \zeta_{i} r_{i}\right)^{2}}\right) \\
& \operatorname{Im}(F R F)=\sum_{i=1}^{n} \frac{1}{k_{i}}\left(\frac{-2 \zeta_{i} r_{i}}{\left(1-r_{i}^{2}\right)^{2}+\left(2 \zeta_{i} r_{i}\right)^{2}}\right)
\end{aligned}
$$

Where $\operatorname{Re}(F R F)$ and $\operatorname{Im}(F R F)$ are real and image values of an eigenvalue, respectively. $n$ is the number of modes ( $n=2$ for the FRF from Fig.10), $k_{i}$ is the modal stiffness of the $i^{\text {th }}$ mode, $r_{i}=$ $\omega / \omega_{n, i}$ is the frequency ratio of the $i^{t h}$ mode, $\omega$ is the excitation frequency and $\omega_{n, i}$ is the modal natural frequency of the $i^{t h}$ mode. The frequencies labelled $\omega_{n, 1}$ and $\omega_{n, 2}$ along the frequency axis in the imaginary part of the direct FRF correspond to the minimum imaginary peaks and provide the two natural frequencies. The damping ratios can be given by half-power points:

$$
\zeta_{1}=\frac{\omega_{4}-\omega_{3}}{2 \omega_{n, 1}} ; \zeta_{2}=\frac{\omega_{6}-\omega_{5}}{2 \omega_{n, 2}}
$$

The negative peak values A and B, identified along the vertical axis of the imaginary part of the direct FRF are used to find the modal stiffness values $k_{1}$ and $k_{2}$, respectively:

$$
k_{1}=\frac{-1}{2 \zeta_{1} A} ; \quad k_{2}=\frac{-1}{2 \zeta_{2} B}
$$

The modal masses can be determined by using the natural frequencies and modal stiffness values:

$$
m_{1}=\frac{k_{1}}{\omega_{n 1}^{2}} \text { and } m_{2}=\frac{k_{2}}{\omega_{n 2}^{2}}
$$

The modal parameters in Y direction can be obtained use the same method. The obtained modal parameters using the peak-picking fitting methods are given in Table 2. The modal parameters are used in the chatter model.

The modal parameters of the machine tool system are then obtained from Fig.10 by the modal fitting technology and shown in Table 2. 

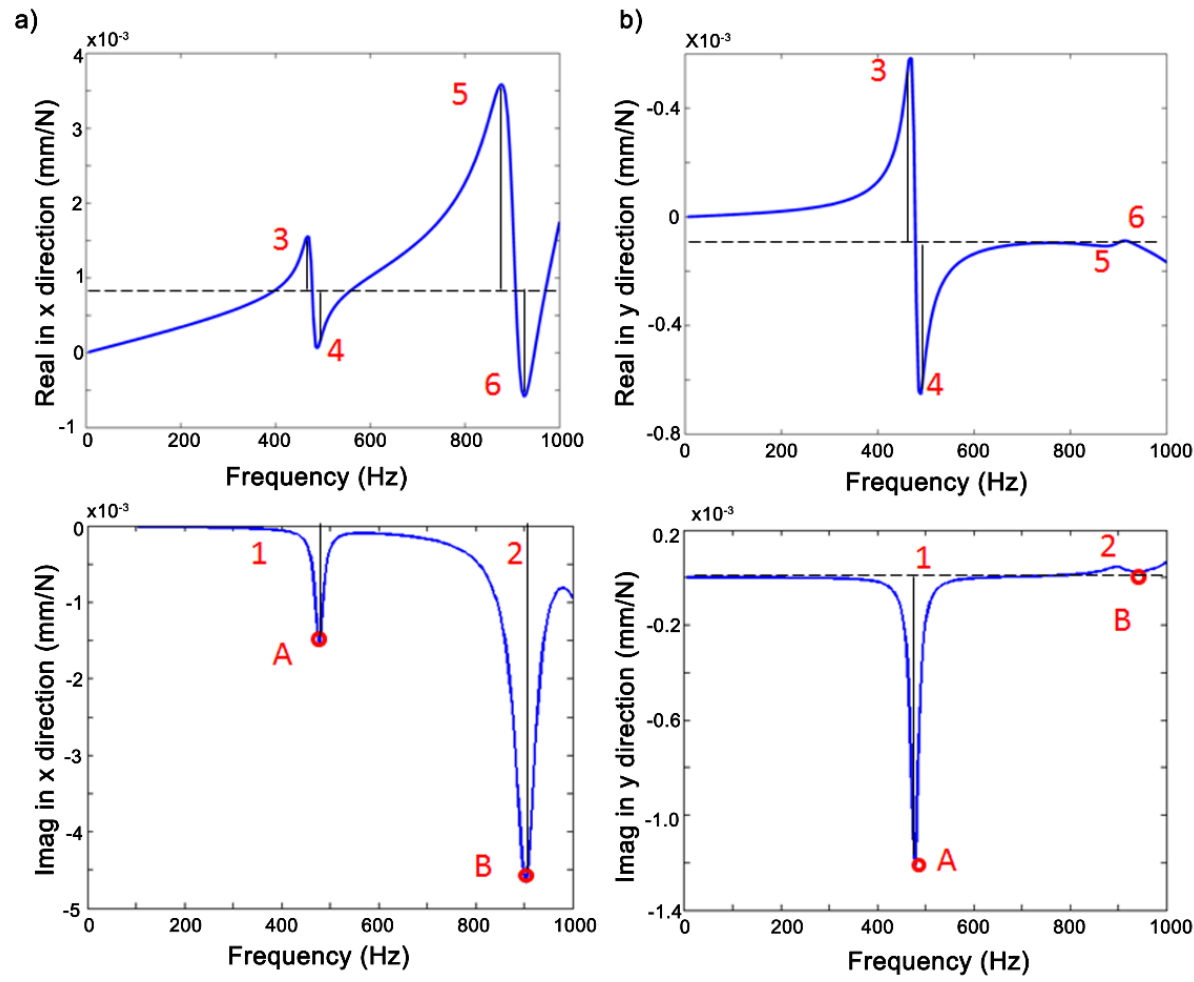

Fig.10 Tool point FRF. a) Real and imaginary parts in $\mathrm{x}$ direction; b) Real and imaginary parts in y direction.

Table 2 Modal parameters obtained by modal fitting

\begin{tabular}{llllll}
\hline & & Stiffness $(\mathrm{N} / \mathrm{mm})$ & Damping ratio & Mass $(\mathrm{Kg})$ & Natural frequency $(\mathrm{Hz})$ \\
\hline \multirow{2}{*}{ X direction } & Model 1 & 22000 & 0.015 & 96.28 & 478 \\
\cline { 2 - 6 } & Model 2 & 61728 & 0.018 & 76.2 & 900 \\
\hline \multirow{2}{*}{ Y direction } & Model 1 & 26500 & 0.0156 & 116 & 478 \\
\cline { 2 - 6 } & Model 2 & $3.125 \mathrm{e} 7$ & 0.016 & $3.8 \mathrm{e} 4$ & 900 \\
\hline
\end{tabular}

The dynamic response of the machine tool system under the action of the cutting forces can be obtained as [21]:

$$
\left\{\begin{array}{l}
M_{x} \ddot{x}(t)+C_{x} \dot{x}(t)+K_{x} x(t)=F_{x}(t) \\
M_{y} \ddot{y}(t)+C_{y} \dot{y}(t)+K_{y} y(t)=F_{y}(t)
\end{array}\right.
$$

Where $F x(\mathrm{t})$ and $F y(\mathrm{t})$ are the dynamic cutting force in $x$ and $y$ directions, respectively. $M_{x}$ and $M_{y}, C_{x}$ and $C_{y}, K_{x}$ and $K_{y}$ are the equivalent mass, equivalent damping and equivalent stiffness in $x$ and $y$ directions, respectively.

The machining chatter vibration can be predicted by Equation (16)

$$
\begin{gathered}
b_{l i m}=\frac{-1}{2 K_{s} \cos (\beta) \operatorname{Re}[F R F] N_{t}^{*}} \\
\frac{f_{c}}{\Omega}=N+\frac{\varepsilon}{2 \pi} \\
\varepsilon=2 \pi-2 \tan ^{-1}\left(\frac{\operatorname{Re}[F R F]}{\operatorname{Im}[F R F]}\right)
\end{gathered}
$$

Where, $b_{\text {lim }}$ is the limiting cutting depth to a void chatter, $N^{*}$ is the average number of teeth in cut, $K_{S}$ is the cutting coefficient, $\beta$ is the force angle, $N$ is the integer number of waves of vibration 
imprinted on the workpiece surface in one revolution, $\varepsilon$ is the phase between the current and previous tool vibration, $f_{c}$ is chatter frequency, $\Omega$ is the spindle rotation speed.

Fig. 11 illustrate the stability lobes diagram, it can be found that with the $10 \mu \mathrm{m} /$ tooth feed rate, when the cutting depth less than $0.2 \mathrm{~mm}$, the machining state is keeping stable with all speed spindle, this region namely static stable region. In some spindle speed when the cutting depth larger than $0.2 \mathrm{~mm}$, if the machine state is also stable, those regions namely dynamic stable region, if not namely unstable region.

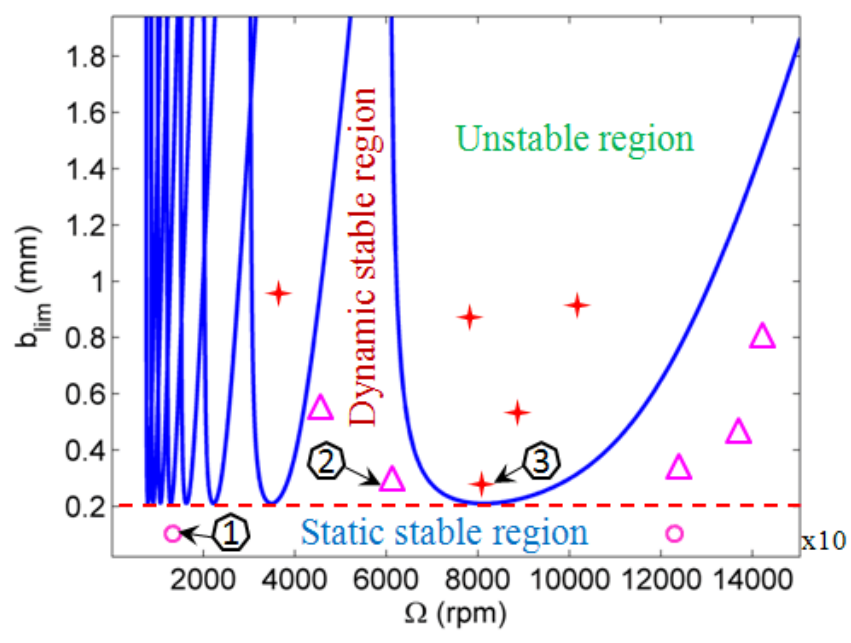

Fig.11 Stability lobes diagram

\subsection{Surface generation simulation}

To indicate the feasibility of the proposed surface generation model and verify the simulation results, the same machining parameters are used in the surface simulation and the machining experiments.

The surface generation simulation of slot micro milling was performed with three sets machining parameters as shown in Table 3. Three sets of machining parameters were chosen to correspond to three machining states as shown in Fig.11. That is, the selection of machining parameters of Set 1, Set 2 and Set 3 is intended to correspond to static stable, dynamic stable and unstable machining state, respectively.

Table 3 Machining parameters

\begin{tabular}{cccc}
\hline Slot milling & Set 1 & Set 2 & Set 3 \\
\hline Spindle speed $(\mathrm{rpm})$ & 15,000 & 60,000 & 80,000 \\
\hline Cutting depth $(\mathrm{mm})$ & 0.1 & 0.3 & 0.3 \\
\hline Feed per tooth $(\mu \mathrm{m})$ & 25 & 10 & 10 \\
\hline
\end{tabular}

Fig.12 gives the tool tip trajectory at each set of machining parameters. It can be found from Fig. 12 a) and b) that tool tip trajectories at the machining parameters of Set 1 and Set 2 are uniform, indicating that the machining process is stable. As for the tool tip trajectory at the machining parameters of Set 3 , irregular fluctuation could be clearly observed (see Fig. 12 c), which means that unstable machining process is being conducted.

In the simulations, the cutting edge of the tool is regarded as a rigid body. The displacement of each point on the cutting edge in each direction is consistent, thus the dynamic trajectory of the whole cutter can be obtained from that of the tool tip. The machined surface can be then generated by the 
superposition of each trajectory of the cutting edge by the $\mathrm{Z}$ map technology.
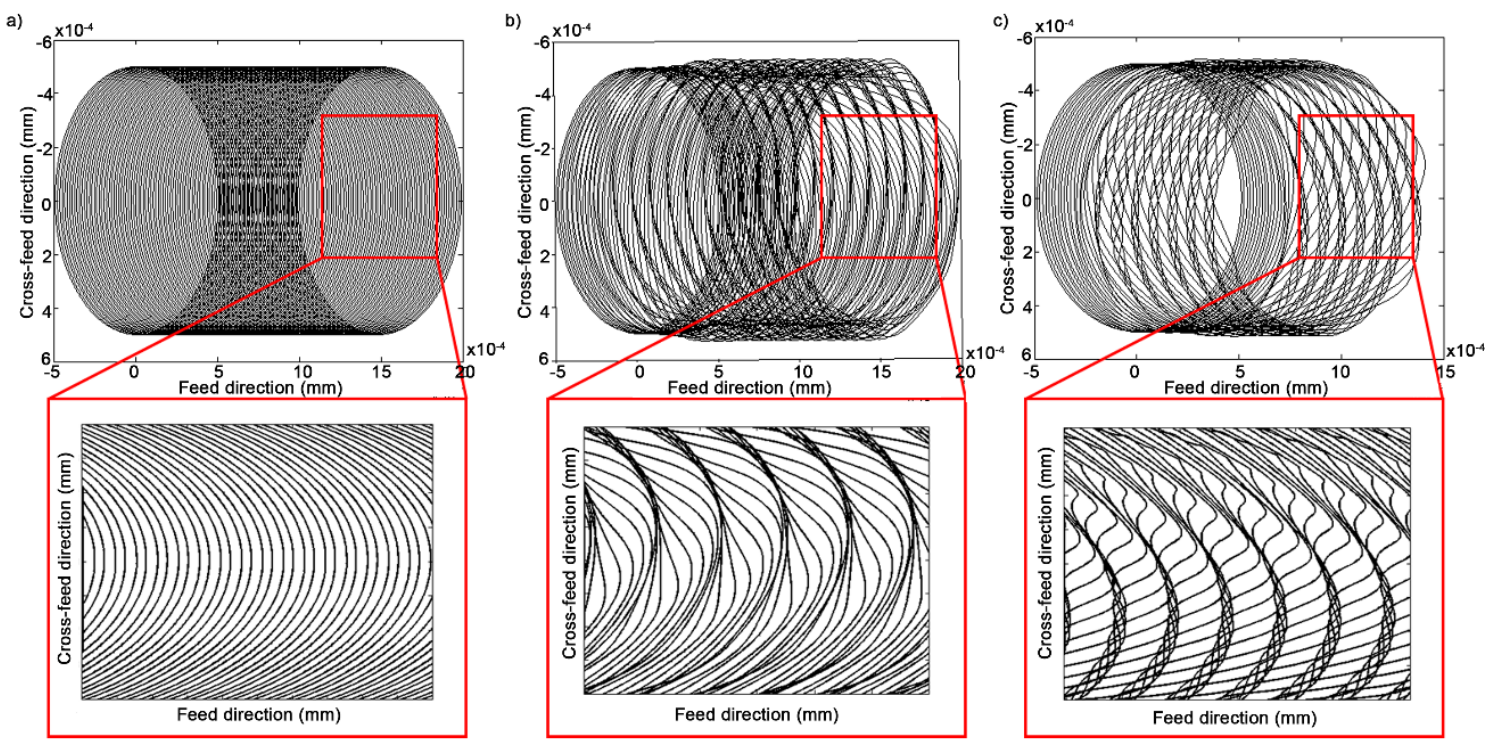

Fig.12 Tool tip trajectories at different spindle speeds: a) 15,000rpm; b) 60,000rpm; c) 80,000rpm

In order to indicate the effects of machining dynamics on the surface topography generation, Figs.13-15 compares the surface topographies obtained from the traditional model which does not consider the machining dynamics and the proposed model at different machining parameters, and the experimental results are also given to verify the superiority of the proposed method.

As shown in Fig.13, when the machining is performed at a spindle speed of 15,000rpm and cutting depth of $0.1 \mathrm{~mm}$, the simulated surface topographies from the traditional and proposed models are nearly identical. Evident and regular tool marks can be clearly found and the surface roughness (RMS) values are about $0.18 \mu \mathrm{m}$. Regular tool marks also can be clearly found on the tested surface, the surface roughness (RMS) values are measured to be $0.19 \mu \mathrm{m}$. Therefore, it can be noted that when the machining process in a static stable region, the traditional model has the same prediction ability with the proposed model, the prediction error of the surface roughness is around $5.2 \%$.

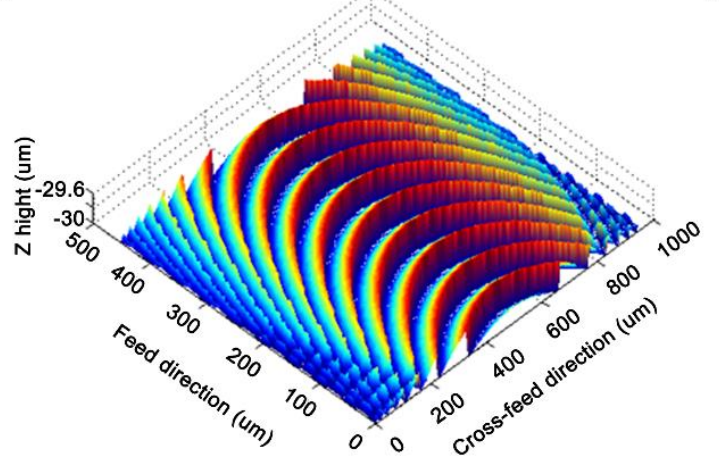

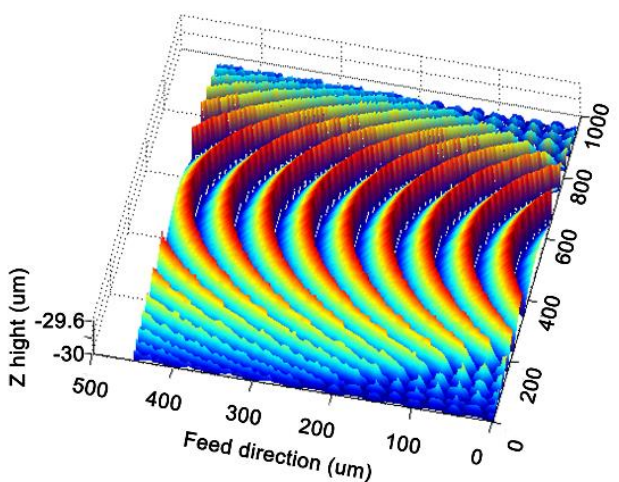


c)
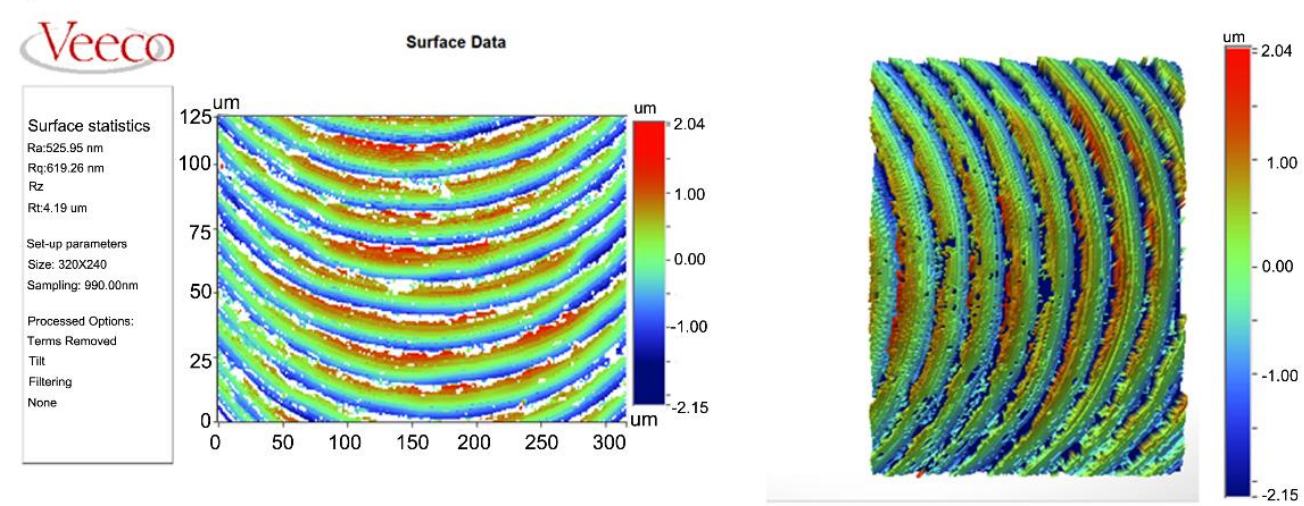

Fig. 13 Surface topographies at spindle speed of 15,000rpm. a) without considering the machining dynamics; b) considering the machining dynamics; c) experimental result.

When the spindle speed and cutting depth change to $60,000 \mathrm{rpm}$ and $0.3 \mathrm{~mm}$, the simulated surface topographies (see Fig.14 a) from traditional model is similar to those given in Fig.13 a). The RMS value is also about $0.18 \mu \mathrm{m}$. Conversely, when considering the effects of machining dynamics on the surface topography, the simulated surface topography has changed. As shown in Fig. 14 b), due to the dynamic response of the machining system, the tool marks have changed with the tool feeding, while regular waviness has been formed on the machined surface and the RMS value has turns to about $0.22 \mu \mathrm{m}$. The difference between surface topographies in Fig.14 a) and b) clearly indicates that the surface generation process has been affected by the dynamic response of the machining system, although stable machining still dominates. The simulation results obtained by the proposed model agree well with the experimental results as shown in Fig. 14 c). The evident waviness and regular tool marks could be clearly detected on the machined surface, which indicates that the machining process belongs to the dynamic region. The surface roughness error predicted by the traditional model is around $25.9 \%$ which is significantly larger than $9.46 \%$ as predicted by the proposed model.
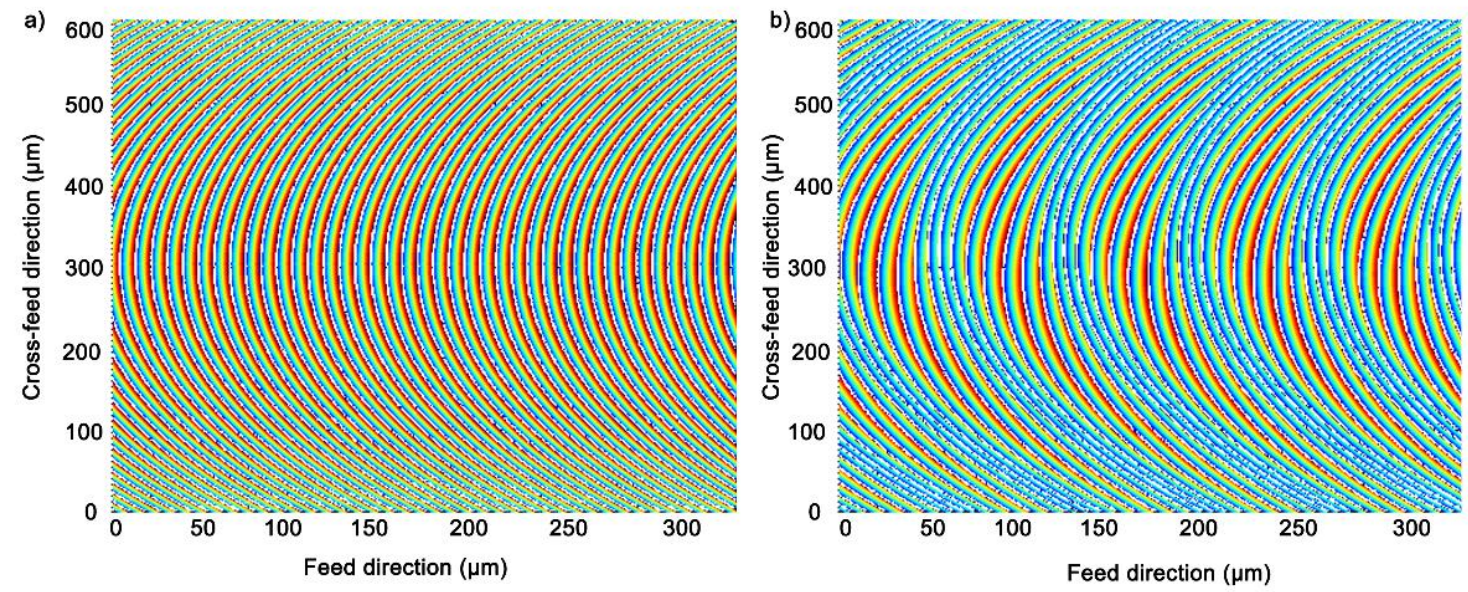
c)

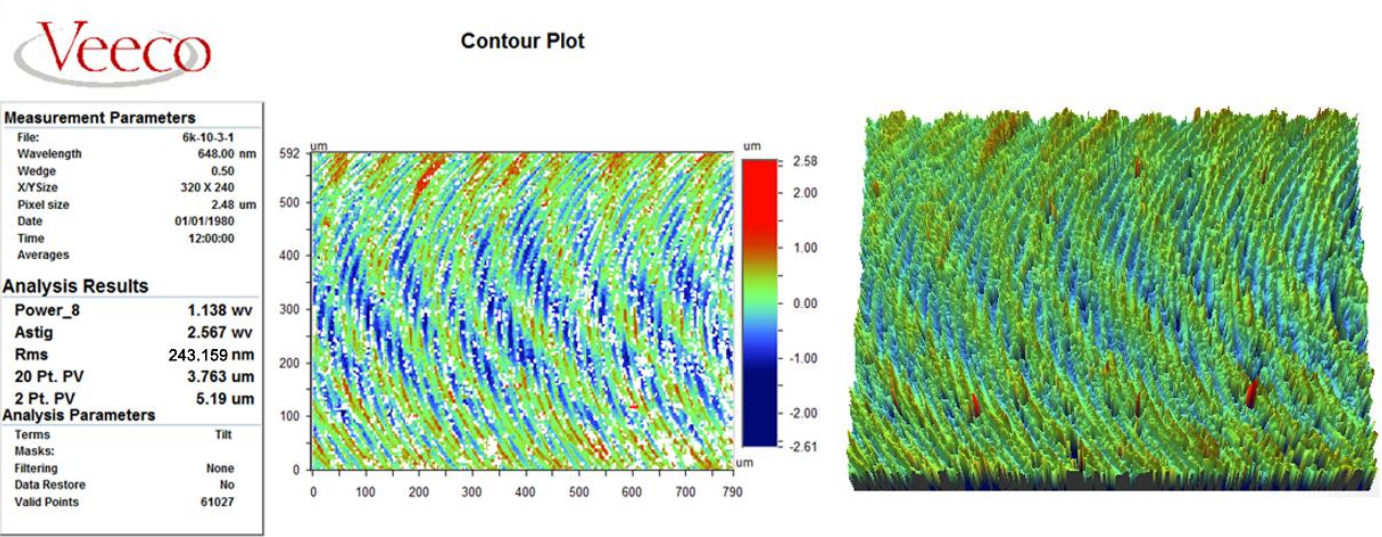

Fig. 14 Simulated surface at spindle speed of 60,000rpm. a) without considering the machining dynamics; b) considering the machining dynamics; c) experimental result.

When the spindle speed and cutting depth increase to $80,000 \mathrm{rpm}$ and $0.3 \mathrm{~mm}$, as shown in Fig.15, distinct surface topographies are obtained. The difference is attributed to the machining dynamics effect. For the case ignoring machining dynamics, regular tool marks can be observed as shown in Fig.15 a). Similar to the surface topographies in Fig.13a) and Fig.14a). The RMS value of about $0.18 \mu \mathrm{m}$ is predicted by the traditional model. However, when considering the machining dynamics, the surface topography has evidently changed. As shown in Fig. 15 b), no regular circular tool marks could be seen on the machined surface, which agrees with the measured surface topography as shown in Fig. $15 \mathrm{c}$ ), and the predicted RMS value becomes $0.536 \mu \mathrm{m}$. The surface roughness error of the traditional model has reached around $65.2 \%$, which indicates that the traditional model is ineffective at higher cutting speed and cutting depth. While the proposed model still provides good surface roughness prediction with an error of around $3.47 \%$.

a)

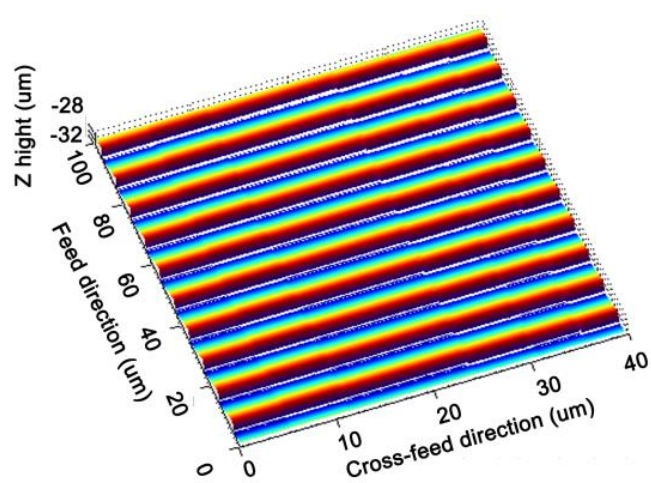

b)

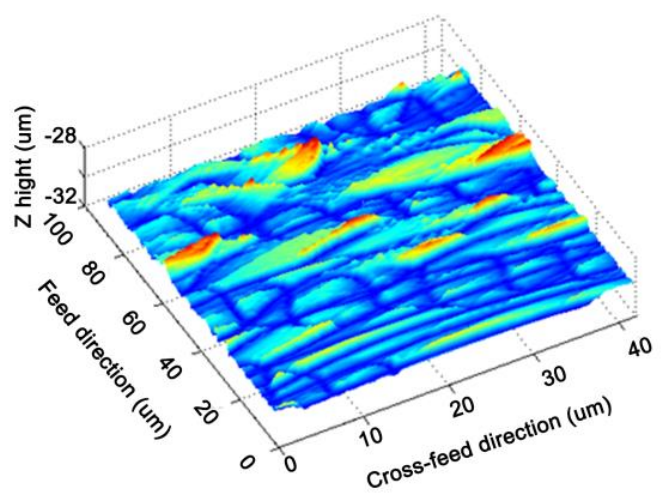


c)

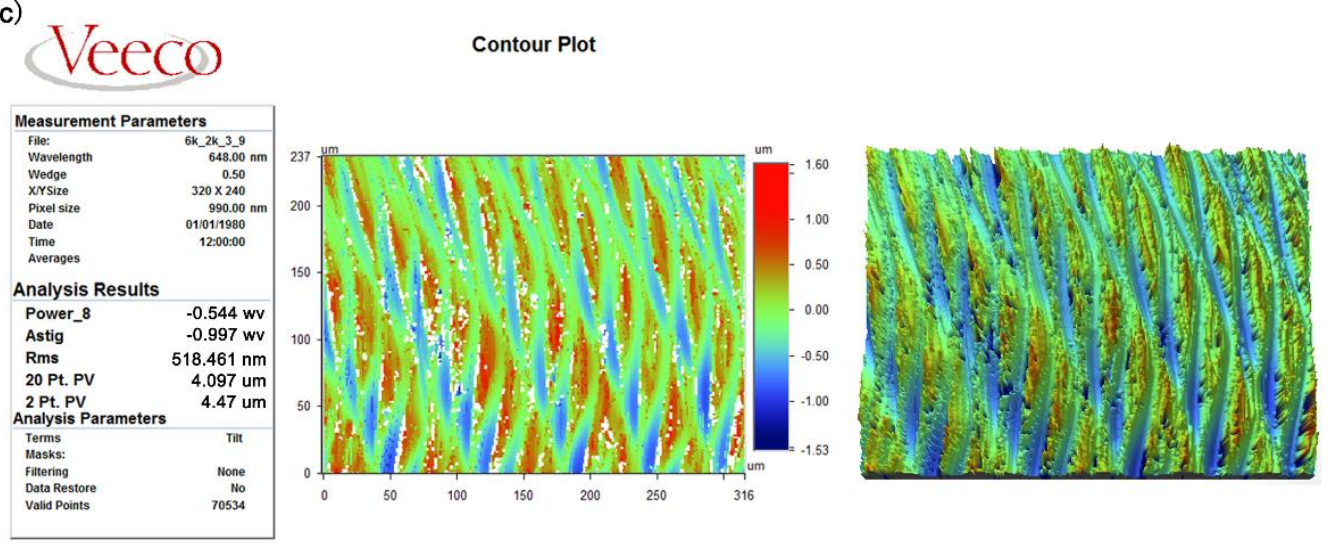

Fig. 15 Simulated surface at spindle speed of 80,000rpm. a) without considering the machining dynamics; b) considering the machining dynamics; c) experimental result.

Comparing Fig.13-15, it can be observed that the measured surface topographies at different machining states have undergone evident change, which agrees well with the simulation results from the proposed model. It can be concluded that the proposed simulation method for surface topography generation can accurately realize the 3D simulation of micro end milling process. Table 4 list the surface roughness RMS values from experiments and simulations. It can be found that the traditional model can be only used when the machining in the static stable region. In the dynamic stable and unstable machining regions, the prediction errors obtained by using traditional model has increased to $25.9 \%$ $(60000 \mathrm{rpm})$ and $65.2 \%(80,000 \mathrm{rpm})$, respectively. Thus, it can be concluded that the traditional model cannot be employed to accurately predict the surface generation. The proposed model can provide accurate surface generation prediction in all machining conditions, with RMS prediction errors being $9.46 \%$ (60000 rpm) and 3.47\% (80,000 rpm), respectively.

Table 4 Comparison of the RMS value between the simulation and experiment results

\begin{tabular}{ccccccc}
\hline & \multicolumn{3}{c}{ Surface roughness $(\mu \mathrm{m})$} & & \multicolumn{2}{c}{ Error $(\%)$} \\
\cline { 2 - 5 } Spindle speed $(\mathrm{rpm})$ & $\begin{array}{c}\text { Traditional } \\
\text { model }\end{array}$ & $\begin{array}{c}\text { Proposed } \\
\text { model }\end{array}$ & Experiment results & & $\begin{array}{c}\text { Traditional } \\
\text { model }\end{array}$ & $\begin{array}{c}\text { Proposed } \\
\text { model }\end{array}$ \\
\hline 15,000 & 0.18 & 0.18 & 0.19 & 5.2 & 5.2 \\
\hline 60,000 & 0.18 & 0.22 & 0.243 & 25.9 & 9.46 \\
\hline 80,000 & 0.18 & 0.536 & 0.518 & 65.2 & 3.47 \\
\hline
\end{tabular}

It should be noted that the proposed surface model is a tool geometry-based model for surface topography generation. It can be applied for the simulation of surface topographies of the machined surface formed with any tool geometry. It is well known that tool wear has great influence on the surface generation. Thus, the tool wear induced changes of tool geometry parameters, such as corner radius and the rake angle, should be considered. In the surface generation model proposed in this work, the influence of tool wear on the surface generation can be included using updated tool geometries either from measurement or a separate tool wear modelling approach.

\section{Conclusion}

In this paper, a novel HMT-based surface generation model is proposed for micro milling. Surface 
generation simulation was conducted and further verified by micro end milling experiments. The following conclusions can be drawn:

1) Based on the HMT technology, a real 3D machined surface generation simulation model is proposed to consider the cutting edge geometry and the machining path. On this basis, the prediction for the surface roughness and surface topography of the micro-milled surface is realized.

2) The influence of machining process on the surface generation is studied by considering the dynamic regenerative effect. The experimental results demonstrate that the proposed simulation method can accurately predict the surface roughness and surface topographies at three different machining states, namely static stable region, dynamic stable region and unstable region.

3) The proposed surface simulation model bridges the gap between surface topography prediction and the practical machining states by considering the effect of the dynamic performance of the machining system, and hence provides a better understanding of the surface generation in micro milling process.

4) The proposed surface simulation model can be applied not only to evaluate the machining stability, but also to predict the roughness of the machined surface.

5) With the consideration of the machining dynamics, the machining stability can be included in the proposed surface model with the specific machining parameters, which can be used for machining quality prediction and the machining parameters selection.

\section{Acknowledgment}

The authors gratefully acknowledge financial support of the Engineering and Physical Sciences Research Council (EP/M020657/1), National Natural Science Foundation of China (Grant No.51505107) and Project (HIT.NSRIF.2017029) supported by Natural Scientific Research Innovation Foundation in Harbin Institute of Technology.

\section{Conflict of interest}

The authors declare that there is no conflict of interest.

\section{Reference:}

[1] Huo, D., Choong, Z., Shi, Y., Hedley, J., and Zhao, Y. Diamond Micro-milling of Lithium Niobate for Sensing applications, Journal of Micromechanics and Microengineering, 2016, 26095005

[2] Huo, D., Lin, C. and Dalgarno, K. An experimental investigation on micro machining of finegrained graphite, International Journal of Advanced Manufacturing Technology, 2014, 72, 943-953

[3] Liu X, DeVor R E, Kapoor S G. Model-based analysis of the surface generation in microendmilling - part I: model development[J]. Journal of manufacturing science and engineering, 2007, 129(3): 453-460.

[4] Jung,W.C., Heo, Y.M., Yoon,G.S., and et al. Micromachining of injection mold inserts for fluidic channel of polymeric biochips. Sensors, 2007; 7: 1643-1654.

[5] Rawool A S, Mitra S K, Kandlikar S G. Numerical simulation of flow through microchannels with designed roughness[J]. Microfluidics and nanofluidics, 2006, 2(3): 215-221.

[6] Govekar, E., et al. "On stability and dynamics of milling at small radial immersion." CIRP AnnalsManufacturing Technology 54.1 (2005): 357-362. 
[7] Long, X-H., B. Balachandran, and B. P. Mann. "Dynamics of milling processes with variable time delays." Nonlinear Dynamics 47.1 (2007): 49-63.

[8] Li Mingzhen, Guojun Zhang, and Yu Huang. "Complete discretization scheme for milling stability prediction." Nonlinear Dynamics 71.1-2 (2013): 187-199.

[9] Afazov, S. M., et al. "Chatter modelling in micro-milling by considering process nonlinearities." International Journal of Machine Tools and Manufacture 56 (2012): 28-38.

[10] Ghali, Sherif. "Constructive solid geometry." Introduction to Geometric Computing (2008): 277283.

[11] Peigne G, Paris H, Brissaud D. A model of milled surface generation for time domain simulation of high-speed cutting $[\mathrm{J}]$. Proceedings of the Institution of Mechanical Engineers, Part B: Journal of Engineering Manufacture, 2003, 217(7): 919-930.

[12] Vogler M P, DeVor R E, Kapoor S G. On the modeling and analysis of machining performance in micro-endmilling, Part I: Surface generation[J]. Journal of Manufacturing Science and Engineering, 2004, 126(4): 685-694.

[13] Sun Y, Liang Y, Du R. Simulation and analysis of surface generation in micromilling[C]//Proceedings of the 6th WSEAS International Conference on Robotics, Control and Manufacturing Technology, Hangzhou, China. 2006: 30-35.

[14] Chen W, Huo D, Sun Y, et al. "Surface Generation Modelling for Micro end Milling Considering the Minimum Chip Thickness and Tool Runout." Procedia CIRP58 (2017): 364-369.

[15] Kouravand S, Imani B M. Developing a surface roughness model for end-milling of microchannel[J]. Machining Science and Technology, 2014, 18(2): 299-321.

[16] Li H, Lai X, Li C, et al. Modelling and experimental analysis of the effects of tool wear, minimum chip thickness and micro tool geometry on the surface roughness in micro-end-milling. Journal of Micromechanics and Microengineering 2007; 18(2): 025006.

[17] Chen W, Teng X, Huo D, et al. An improved cutting force model for micro milling considering machining dynamics[J]. The International Journal of Advanced Manufacturing Technology, 2017: $1-12$.

[18] Huo D, Chen W, Teng X, et al. Modeling the Influence of Tool Deflection on Cutting Force and Surface Generation in Micro-Milling[J]. Micromachines, 2017, 8(6): 188.

[19] G. Chen, Y. Liang, Y. Sun et al., "Volumetric error modeling and sensitivity analysis for designing a five-axis ultra-precision machine tool," The International Journal of Advanced Manufacturing Technology, vol. 68, no. 9-12, pp. 2525-2534, 2013.

[20] Schmitz, Tony L., and K. Scott Smith. Machining dynamics: frequency response to improved productivity. Springer Science \& Business Media, 2008.

[21] Altintas, Y., and D. Montgomery. "Mechanism of cutting force and surface generation in dynamic milling." Journal of Engineering for Industry 113.2 (1991): 160-168 